\title{
Acetylation Modification Improves Immunoregulatory Effect of Polysaccharide from Seeds of Plantago asiatica $\mathbf{L}$.
}

\author{
Le-Ming Jiang $(\mathbb{D}$, Shao-Ping Nie $\mathbb{D}$, Dan-Fei Huang, Zhi-Hong Fu, and Ming-Yong Xie \\ State Key Laboratory of Food Science and Technology, Nanchang University, Nanchang 330047, China \\ Correspondence should be addressed to Shao-Ping Nie; spnie@ncu.edu.cn and Ming-Yong Xie; myxie@ncu.edu.cn
}

Received 17 October 2017; Revised 13 December 2017; Accepted 8 January 2018; Published 13 February 2018

Academic Editor: Ji Kang

Copyright (C) 2018 Le-Ming Jiang et al. This is an open access article distributed under the Creative Commons Attribution License, which permits unrestricted use, distribution, and reproduction in any medium, provided the original work is properly cited.

\begin{abstract}
The current study was conducted to investigate effects of acetylated Plantago asiatica L. polysaccharides (Ac-PLCPs) on their immunoregulatory activities in bone-marrow-derived dendritic cells (DCs) model. Influences of natural Plantago asiatica L. crude polysaccharide (PLCP) and Ac-PLCPs on inducing phenotypic and functional maturation on DCs were determined. The results showed that Ac-PLCPs with degree of substitution (DS) from 0.06 to 0.1 could not only stimulate the expression of surface molecules major histocompatibility complex class II (MHC II), cluster of differentiation 86 (CD86), and CD80 on DCs $(P<0.01)$ but also increase the secretion of cytokine IL-12p70 $(P<0.01)$. The endocytosis activity of DCs was attenuated by Ac-PLCPs treatment $(P<0.01)$, while the mRNA expressions of chemokine receptors CCR7 and CXCR4 in DCs were significantly increased $(P<0.01)$. Besides, DCs treated with the Ac-PLCPs showed extremely strong T cell proliferation stimulating activity $(P<0.01)$. These data showed that Ac-PLCPs had higher maturation-stimulating activity on DCs than PLCP, which indicated that acetylation modification improved the immunoregulatory effect of PLCP.
\end{abstract}

\section{Introduction}

Bioactive polysaccharides have attracted extensive attention worldwide, and they are now considered as one functional component of traditional medicine. A great deal of researches indicate that bioactivity of polysaccharide is closely correlated with various structural parameters, such as molecular weight, monosaccharide composition, branches, conformation, and substituent group. Recently, a growing body of research implicates that appropriate structure modification can improve bioactivity of polysaccharide [1]. So far, lots of chemical modifications have been applied to modulate physicochemical or biological properties of polysaccharides, such as carboxymethylation [2,3], sulfation [4], acetylation [5], phosphorylation [6], and oxidation [7] modification.

Acetyl content in some natural polysaccharides has been demonstrated to play an important role in their bioactivities. For example, the lymphocyte proliferation stimulating activity of polysaccharide from Dendrobium nobile mainly depended on the acetyl groups $[8,9]$. Acetylated Ulva pertusa [10], Laminaria japonica polysaccharide [11], and Enteromorpha linza polysaccharide [12] have higher antioxidant activity. Acetylated Grifola frondosa polysaccharide peptides can inhibit glioma C6 cell line in vitro, and the combination treatment of cyclophosphamide and the acetylated polysaccharide peptides can significantly decrease total sialic acid levels in tumor-bear mice [13]. Acetyl fucoidan from Cladosiphon okamuranus could activate murine macrophage cell line, RAW 264.7 cells, through membrane receptor, tolllike receptor 4 (TLR4), cluster of differentiation 14 (CD14), and scavenger receptor class A [14].

Psyllium is a common name used for the plant genus Plantago. In western countries, dietary fiber from psyllium has been used extensively as both pharmacological supplements and food ingredients. Ripe seeds of Plantago asiatica L. are a traditional medicine used as antipyretic, diuretic, and expectorant treatment in China. Our previous study showed that polysaccharide from seeds of Plantago asiatica L. was a high branched heteroxylan consisting of $\beta$-1,4-linked Xylp backbone with side chains attached to O-2 or O-3 position. And its side chains consist of $\beta$-T-linked Xylp, $\alpha$ T-linked Araf, $\alpha$-T-linked GlcA $p, \beta$-Xylp-(1-3)- $\alpha$-Araf, and

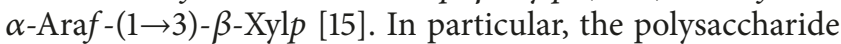
was a potential immune adjuvant since it could promote 
maturation of mouse bone-marrow-derived dendritic cells in vitro [16].

In the present study, Plantago asiatica L. crude polysaccharide (PLCP) were acetylated using acetic anhydride as acetylation reagent, and bioactivities of the acetylated polysaccharides (Ac-PLCP) were investigated on dendritic cells model.

\section{Materials and Methods}

2.1. Preparation of Acetylated Plantago asiatica L. Polysaccharides. PLCP was prepared as previously described [17]. Briefly, water-soluble polysaccharide was extracted from the seeds of Plantago asiatica L. by boiling water for $2 \mathrm{~h}$ and precipitated with $80 \%(\mathrm{v} / \mathrm{v})$ ethanol. Then the polysaccharide was deproteinised with sevage reagent, a mixture of chloroform and n-butyl alcohol at a ratio of $4: 1(\mathrm{v} / \mathrm{v})$, dialysed against double distilled water for $48 \mathrm{~h}$ (Mw cut-off, $8000-14,000 \mathrm{kDa})$, and obtained by freeze-drying.

Acetylation modification of PLCP was carried out using acetic anhydride as acetylation reagent [18]. One hundred milligram PLCP was firstly dissolved in $10 \mathrm{~mL}$ distilled water ( $\mathrm{pH}$ 9.0). The solution was maintained at $30^{\circ} \mathrm{C}$ with continuous stirring. Acetic anhydride (400-2000 $\mu \mathrm{L})$ was added to the solution dropwise within $2 \mathrm{~h}$ ( $\mathrm{pH} 8.5-9.0)$. After the modification reaction, the mixture was neutralized with $1 \mathrm{M}$ $\mathrm{HCl}$ and dialysed against double distilled water for $48 \mathrm{~h}(\mathrm{Mw}$ cut-off: $8000-14,000 \mathrm{kDa}$ ). The acetylated PLCP (AC-PLCP) was obtained by freeze-drying.

The degrees of substitution (DS) of the acetyl group in the Ac-PLCPs were determined as described [19]. Briefly, $40 \mathrm{mg}$ Ac-PLCP was dissolved in $20 \mathrm{~mL} 0.01 \mathrm{M} \mathrm{NaOH}$. The solution was maintained at $50^{\circ} \mathrm{C}$ for $2 \mathrm{~h}$, then excess $\mathrm{NaOH}$ was backtitrated with $0.01 \mathrm{M} \mathrm{HCl}$ using phenolphthalein indicator. Four Ac-PLPCs with gradient degrees of substitution, that is, DS of 0.027 (Ac-PLCP I), 0.059 (Ac-PLCP II), 0.082 (Ac-PLCP III), and 0.11 (Ac-PLCP IV), were selected to investigate the immune regulatory activity in dendritic cell model.

In addition, the bacterial endotoxins in PLCP and AcPLCPs were determined using tachypleus amebocyte lysate test kit (Chinese Horseshoe Crab Reagent Manufactory, Co., Fujian Province, China). The quantity of endotoxin was estimated to be $\leq 0.015$ endotoxin unit (EU) per $\mathrm{mg}$ in all polysaccharide samples.

2.2. Animals. 4- to 6-week-old BALB/c $\left(\mathrm{H}-2 \mathrm{~K}^{\mathrm{d}}\right.$ and $\left.\mathrm{I}-\mathrm{A}^{\mathrm{d}}\right)$ and $\mathrm{C} 57 \mathrm{BL} / 6\left(\mathrm{H}-2 \mathrm{~K}^{\mathrm{d}}\right.$ and $\left.\mathrm{I}-\mathrm{A}^{\mathrm{d}}\right)$ mice were purchased from Hunan SJA Laboratory Animal, Co. (Hunan Province, China). All animals were kept according to the Care and Use of Laboratory Animals Guidelines published by the United States National Institute of Health (NIH Publication 85-23, 1996). All experimental procedures were approved by the Animal Ethnics Committee, Nanchang University.

2.3. Generation of Bone-Marrow-Derived Dendritic Cells (DCs). Bone marrow of BALB/c mice was sacrificed to obtain dendritic cells. According to our previous report [16], bonemarrow cells were flushed out with RPMI 1640 medium, then recombinant murine granulocyte macrophage-colony stimulating factor (rmGM-CSF, $10 \mathrm{ng} / \mathrm{mL}$ ) combined with recombinant murine interleukin-4 (rmIL-4, $10 \mathrm{ng} / \mathrm{mL}$, both from $R \& D$ system) was used to generate immature dendritic cells.

\subsection{Determination of Phenotypic and Functional Maturation} of DCs. Immature DCs were stimulated with the PLCP or Ac-PLCPs $(100 \mu \mathrm{g} / \mathrm{mL})$ for $48 \mathrm{~h}$, and then the phenotypic and functional maturation of DCs were determined according to the methods reported previously [20].

Briefly, the cells were collected and incubated with FITCconjugated monoclonal antibody [anti-major histocompatibility complex (MHC) class II, anti-CD80 (B7-1), and antiCD86 (B7-2), eBioscience] for $1 \mathrm{~h}$, and then the mean fluorescence intensity was determined by using FACSCalibur flow cytometer (BD Biosciences, USA). The endocytosis activity of DCs was also determined by flow cytometry using FITCdextran (40,000 Da, Sigma). The quantity of the cytokine IL-12p70 in the culture supernatant was measured using Mouse IL-12p70 Enzyme Immunoassay kit (Wuhan Boster Biological Technology, China).

The migration activity of DCs was investigated by determining the mRNA expression of chemokine mRNA of CCR7 and CXCR4. Total RNA was extracted using Trizol Reagent (Invitrogen, Carlsbad, CA, USA). Then cDNA was synthesized using RevertAid ${ }^{\text {тм }}$ First Strand cDNA Synthesis Kit (Thermo Scientific, Maryland, USA). PCR products were analyzed by GoldView-agarose gel electrophoresis on $2 \%(\mathrm{w} / \mathrm{v})$ agarose gel and visualized under UV light. The signals of target genes were measured by scanning densitometry and normalized to $\beta$-actin using Quantity One software. The forward and reverse PCR primers used for CCR7 were $5^{\prime}$-GCCTTCCTGTGTGATTTCTACAG- $3^{\prime}$ and 5-TCACCTTCTCTCCTTTCTGTCAC-3' ${ }^{\prime}$; for CXCR4 were $5^{\prime}$-TGTTGCCATGGAACCGATCA-3' and $5^{\prime}$-GGATCCAGACGCCCACATAG- $3^{\prime}$; for $\beta$-actin were $5^{\prime}$-TGGCACCACACCTTCTACAATG- $3^{\prime}$ and $5^{\prime}$-CCTGCTTGCTGATCCACATCTG-3', respectively. Condition of reverse transcription system was $91^{\circ} \mathrm{C}$ for $5 \mathrm{~min}, 4^{\circ} \mathrm{C}$ for $5 \mathrm{~min}, 30^{\circ} \mathrm{C}$ for $10 \mathrm{~min}, 42^{\circ} \mathrm{C}$ for $30 \mathrm{~min}, 99^{\circ} \mathrm{C}$ for $5 \mathrm{~min}$, and $4^{\circ} \mathrm{C}$ for $5 \mathrm{~min}$. Condition of PCR system was initial denaturation at $94^{\circ} \mathrm{C} \times 3 \mathrm{~min}$, followed by 25 cycles of denaturation at $94^{\circ} \mathrm{C}$ $\times 30 \mathrm{~s}$, annealing at $60^{\circ} \mathrm{C} \times 30 \mathrm{~s}$, extension at $72^{\circ} \mathrm{C} \times 2 \mathrm{~min}$, and final extension at $72^{\circ} \mathrm{C}$ for $10 \mathrm{~min}$.

Besides, splenic $\mathrm{T}$ lymphocytes were purified from C57BL/6 $\left(\mathrm{H}-2 \mathrm{~K}^{\mathrm{d}}\right.$ and $\left.\mathrm{I}-\mathrm{A}^{\mathrm{d}}\right)$ mice using Pan T Cell Isolation kit II (Miltenyi Biotec, Inc., Auburn, USA) and then cocultured with DCs for another $48 \mathrm{~h}$. The proliferation of $\mathrm{T}$ cells was determined using MTT Cell Proliferation and Cytotoxicity Assay kit (Beyotime, Shanghai, China).

2.5. Statistical Analysis. Results data were analyzed using SPSS statistical software (version 17.0) and expressed as mean \pm standard deviation (SD) of the indicated number of experiments. The statistical significance was estimated using a Student's $t$-test. $P<0.05$ and $P<0.01$ were considered as statistically significant and highly significant, respectively. 


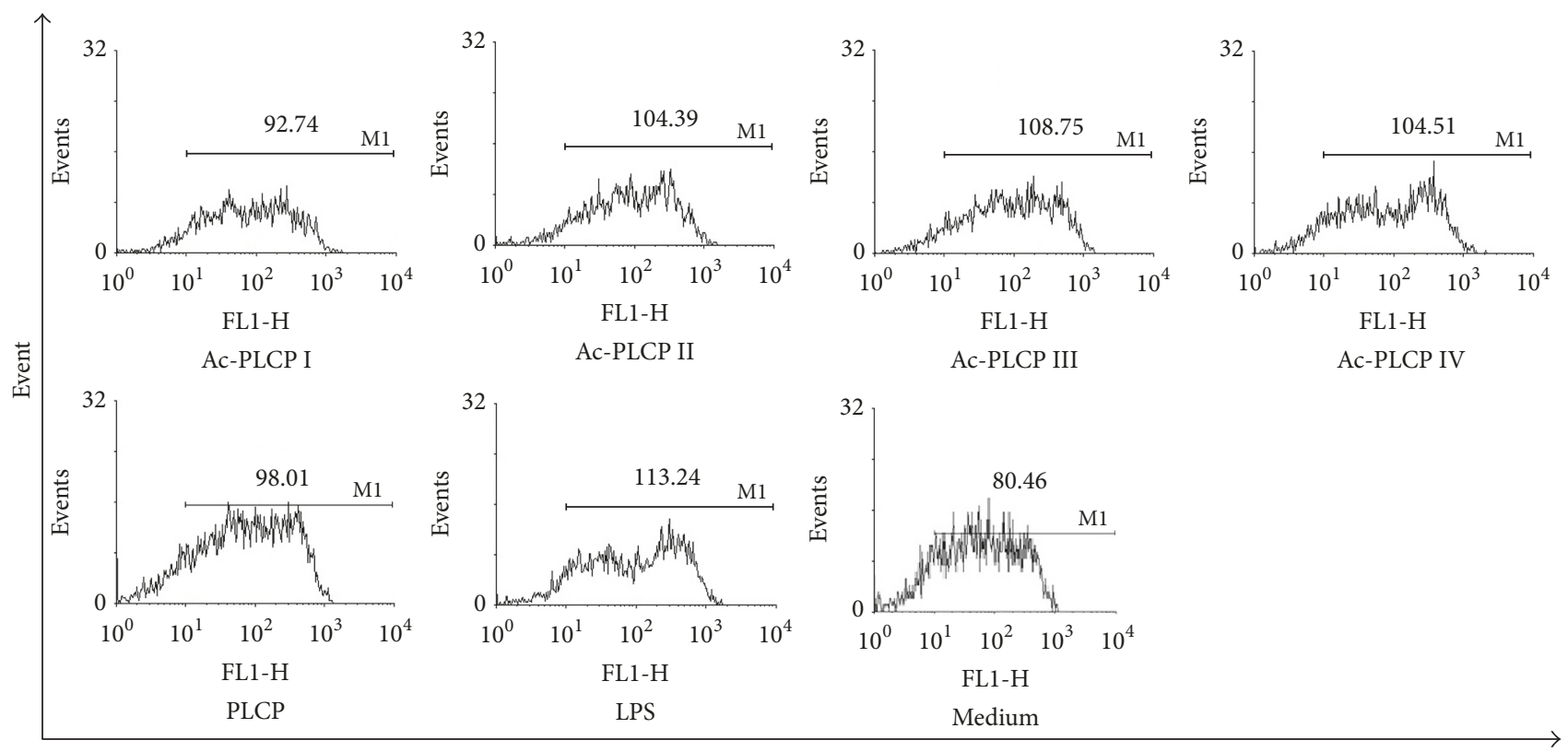

FITC-MHC II

(a)

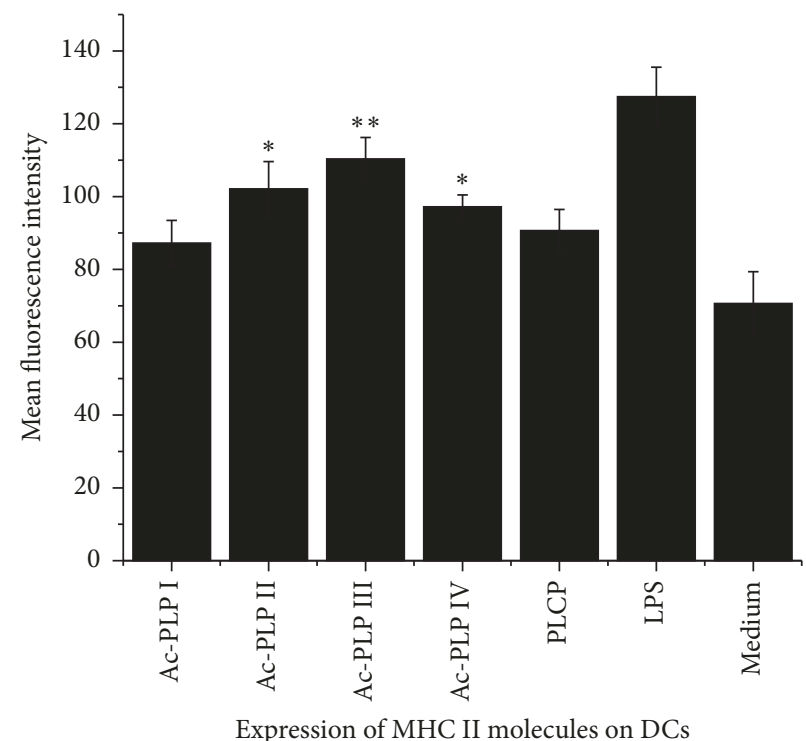

(b)

FIGURE 1: Ac-PLCP increased the expression of MHC II molecules on DCs. Immature DCs were treated with $100 \mu \mathrm{g} / \mathrm{mL}$ PLCP or Ac-PLCPs for $48 \mathrm{~h}$. Then, the expression levels of MHC II molecules were determined by flow cytometry. The data were shown as mean fluorescence intensity (MFI). Compared with medium group, DCs treated with PLCP expressed higher level of MHC II molecules. While compared with the PLCP group, significant increases in MFI were observed in Ac-PLCP II, III, and IV groups. The results shown were from one representative experiment of three independent experiments performed. ${ }^{*} P<0.05$ compared to the PLCP group; ${ }^{* *} P<0.01$ compared to the PLCP group.

\section{Results}

3.1. Ac-PLCP Enhanced Expression of MHC II, CD80, and CD86 Molecules in DCs. Immune responses were initiated through the specific recognition of antigens by lymphocytes, and this progress required stable adhesion of the $\mathrm{T}$ cells to the antigen-presenting cells (APCs), efficient antigen presentation, and transduction of signals from the cell surface to the nucleus of $\mathrm{T}$ cell. In particular, $\mathrm{T}$ cells could only recognize and respond to peptide antigens bound to and displayed by MHC molecules. Flow cytometry was applied to determine the expression of MHC II on DCs, and the data were shown as mean fluorescence intensity (MFI) in Figure 1. After incubation with PLCP, the expression of MHC II on DCs was increased from 80.46 to 98.01. Meanwhile, the expressions of MHC II in Ac-PLCP II, III, and IV groups were 104.39 $(P<0.05), 108.75(P<0.01)$, and $104.51(P<0.05)$, respectively, which were significantly higher than that of PLCP 


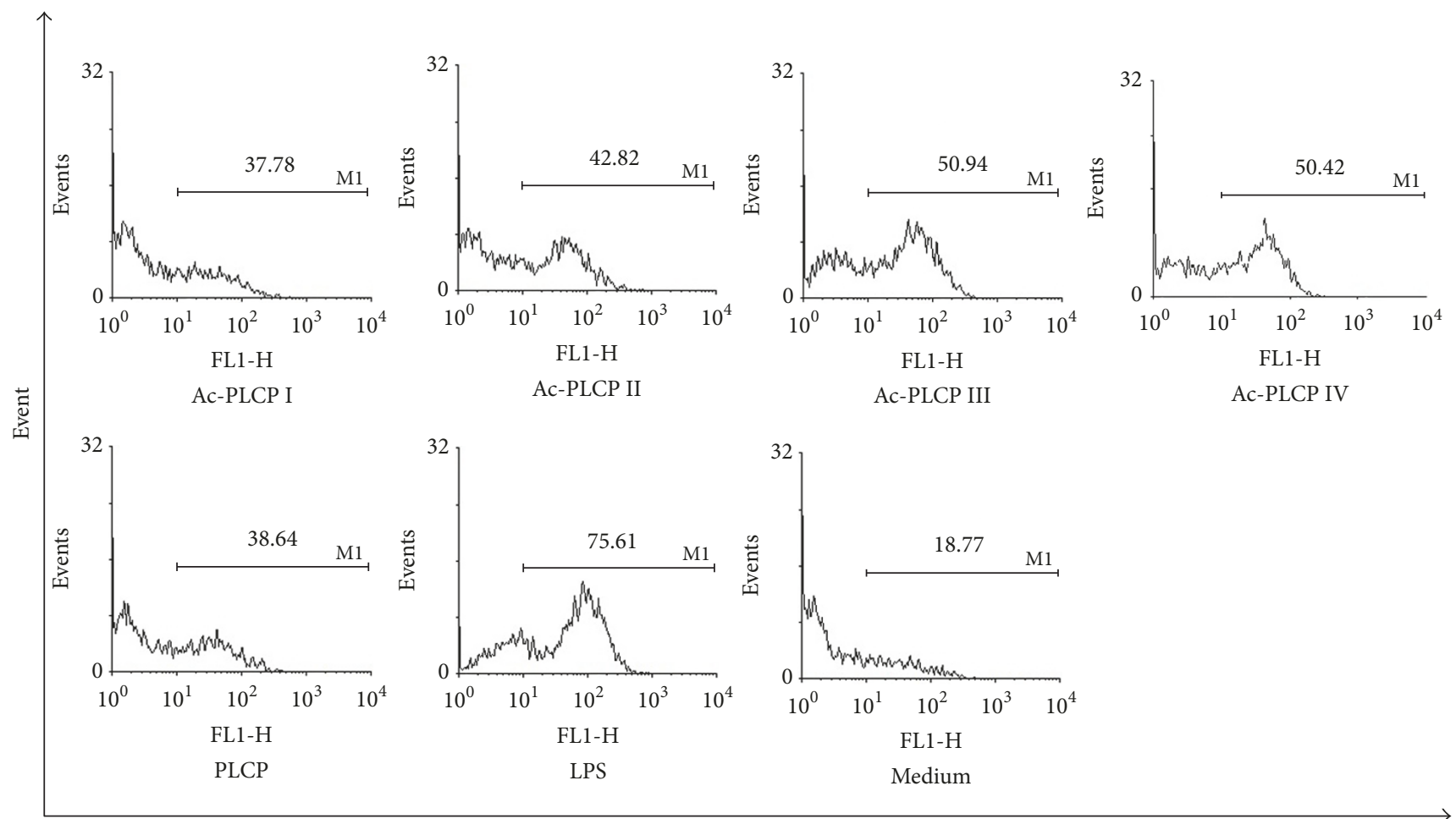

(a)

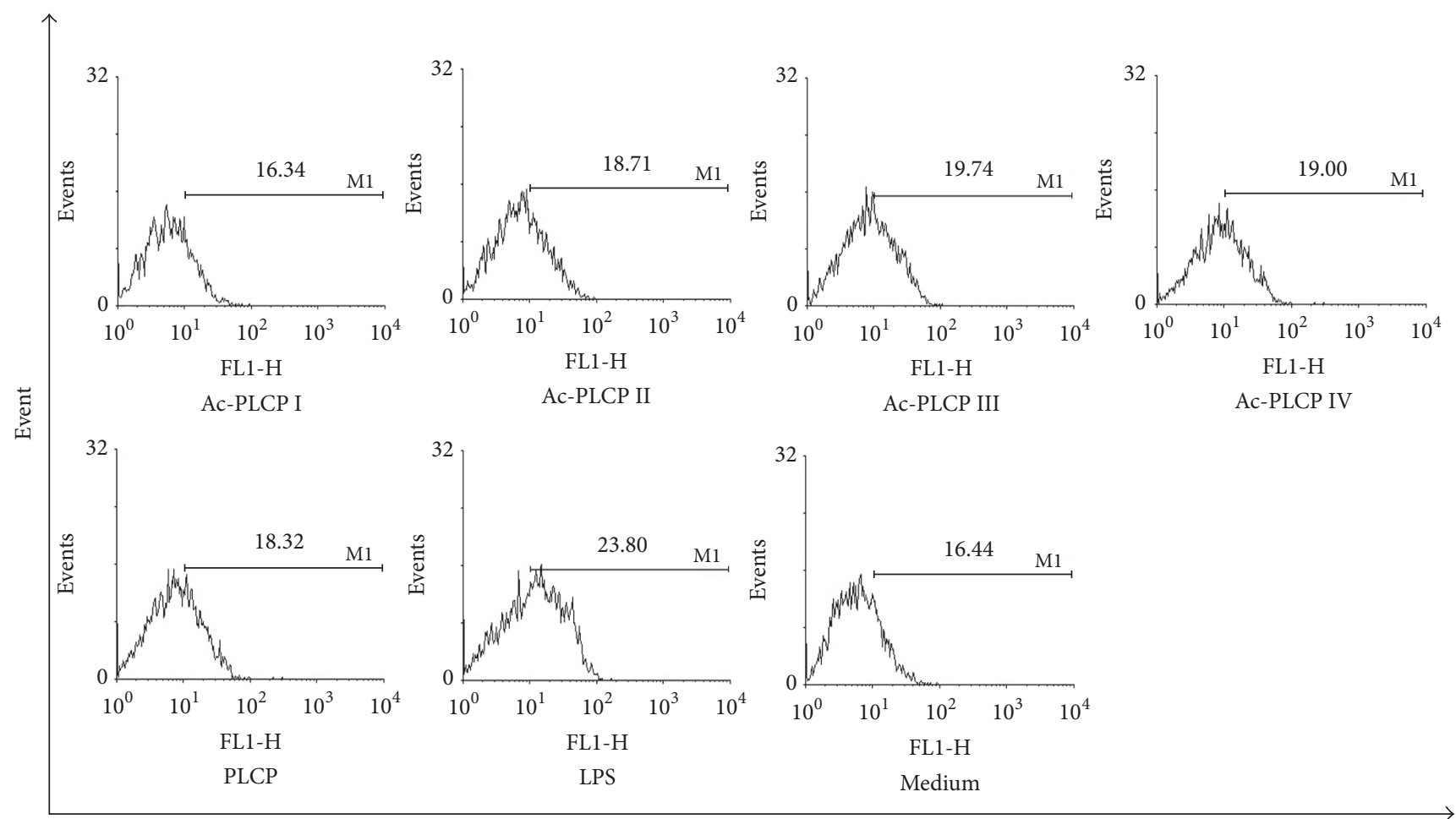

FITC-CD80

(b)

Figure 2: Continued. 


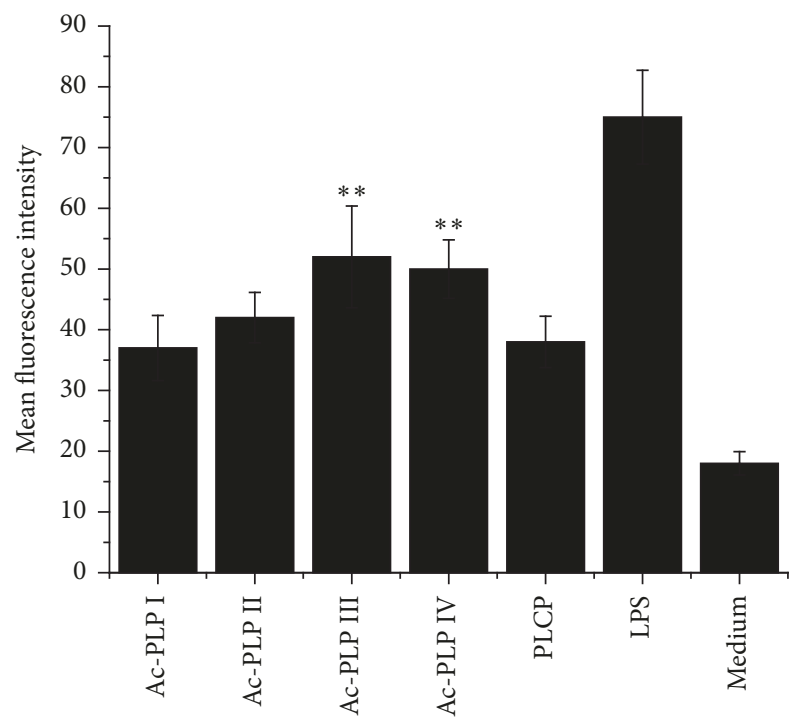

Expression of CD86 molecules on DCs

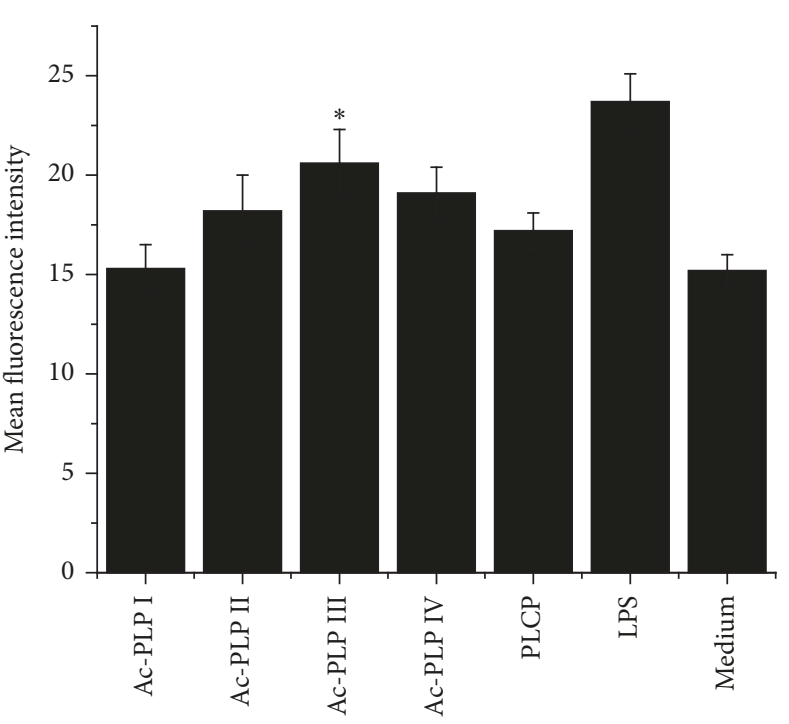

Expression of CD80 molecules on DCs

(c)

Figure 2: Ac-PLCP increased the expression of costimulating molecules on DC. DCs treated with Ac-PLCPs III and IV expressed a higher level of CD86 (a) and CD80 (b) molecules than PLCP group. The results shown were from one representative experiment of three independent experiments performed. ${ }^{*} \mathrm{P}<0.05$ compared to the PLCP group; ${ }^{* *} \mathrm{P}<0.01$ compared to the PLCP group.

group. Costimulating molecules are necessary to ensure an effective immune response. The expressions of CD86 and CD80 costimulating molecules were also measured using flow cytometry. As shown in Figure 2(a), the expression levels of CD86 in DCs in Ac-PLCP I, II, III, and IV groups were $37.78,42.82,50.94$, and 50.42 , respectively, which were much higher than that in the control group (18.77) $(P<0.01)$. In particular, the expression levels of CD86 in Ac-PLCPs III and IV were significantly higher than PLCP group $(P<0.01)$. As shown in Figure 2(b), the expression of CD80 molecules was notably stimulated by Ac-PLCP III (19.74) compared with PLCP group $(16.44)(P<0.05)$.

\subsection{Ac-PLCP Stimulated Secretion of IL-12p70 in DCs.} Beyond interaction with $\mathrm{T}$ cells through surface molecules, DCs also regulate the immune response by secreting cytokines. As shown in Figure 3, PLCP increased the secretion of IL-12p70 in DCs from $20.38 \mathrm{pg} / \mathrm{mL}$ (control group) to $49.97 \mathrm{pg} / \mathrm{mL}$ (PLCP group), while the cytokine levels of IL-12p70 in the acetylated polysaccharides-treated groups were $33.11 \mathrm{pg} / \mathrm{mL}$ (Ac-PLCP I), $60.51 \mathrm{pg} / \mathrm{mL}$ (Ac-PLCP II), $96.43 \mathrm{pg} / \mathrm{mL}$ (Ac-PLCP III), and $81.06 \mathrm{pg} / \mathrm{mL}$ (Ac-PLCP IV), respectively. Compared with PLCP group, Ac-PLCP III and Ac-PLCP IV groups were significantly enhanced $(P<0.01)$.

3.3. Ac-PLCP Attenuated the Endocytosis of DCs. Although DCs are found in almost all tissues in the body, most of the cells exist in immature states. The immature DCs are not professional in antigen peptide presenting but antigen uptaking. However, once the immature DCs receive mature signal, they would develop maturation and transform from antigen-uptake cells into antigen-presenting cells. As shown in Figure 4, DCs in the control group (medium, $37^{\circ} \mathrm{C}$ ) showed

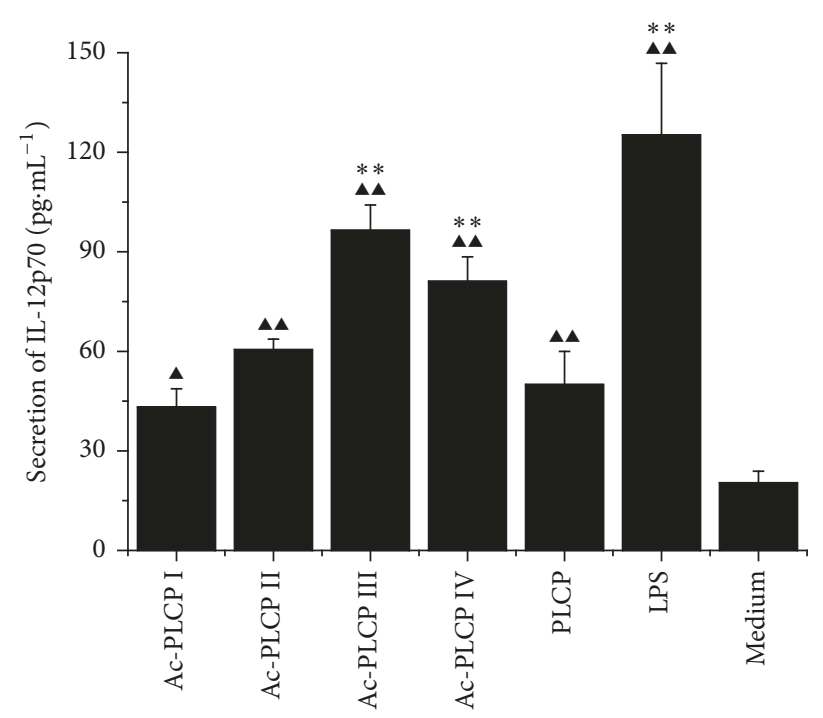

FIgURE 3: Ac-PLCP enhanced the secretion of IL-12p70 cytokine on DCs. Quantity of IL-12p70 in the culture supernatant was determined by ELISA method. Compared with PLCP group, the secretion of DCs in the Ac-PLCPs III and IV was significantly higher than that in PLCP group. ${ }^{* *} P<0.01$ compared to the PLCP group; ${ }^{\wedge} P<0.05$ compared to the medium group; ${ }^{\wedge} P<0.01$ compared to the medium group.

a dramatically high uptake of FITC-dextran. Compared with the control group, DCs in the PLCP and Ac-PLCP groups exhibited an attenuated endocytosis. Particularly, the MFI determined in the Ac-PLCP III (55.17) and IV groups (56.28) was significantly lower than PLCP group $(67.60)(P<0.01)$. 


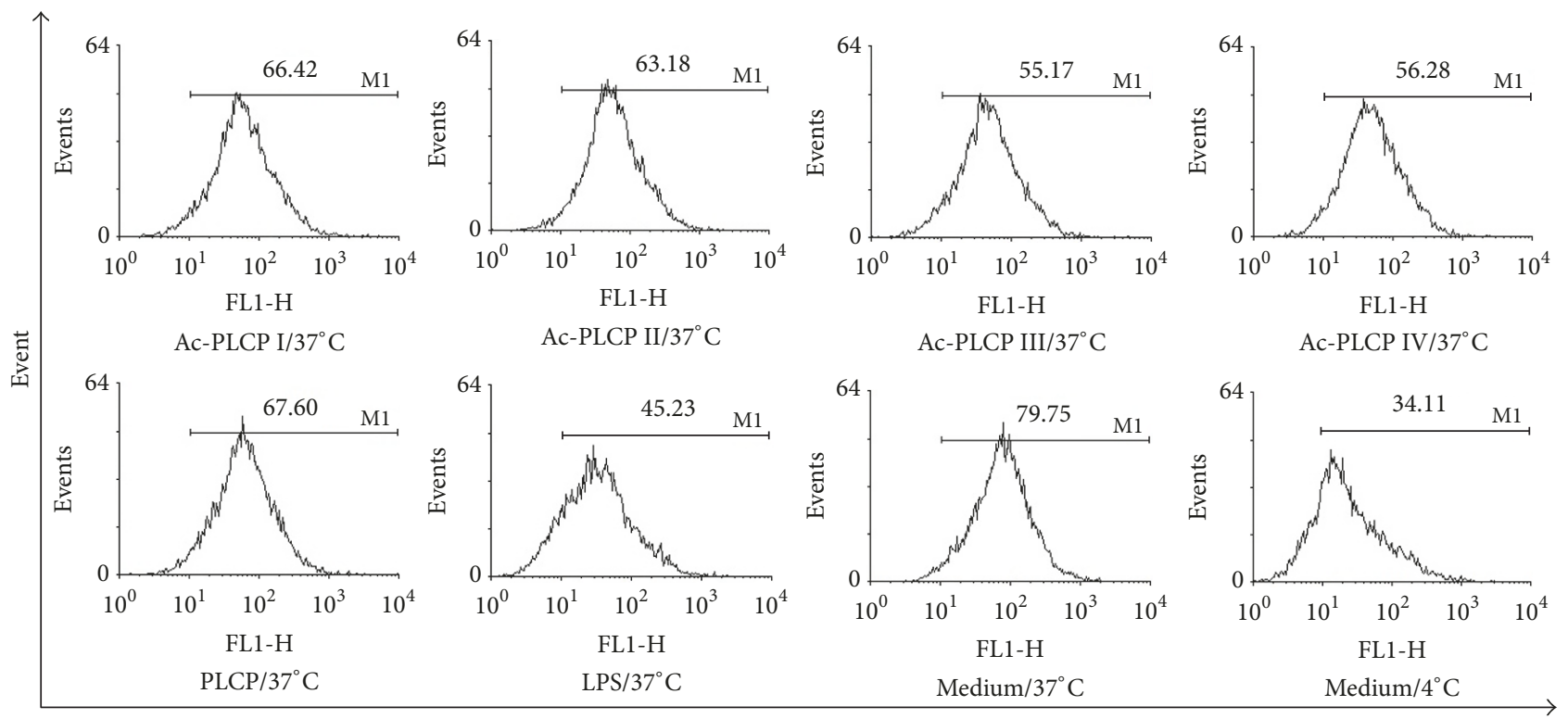

FITC-Dextran

(a)

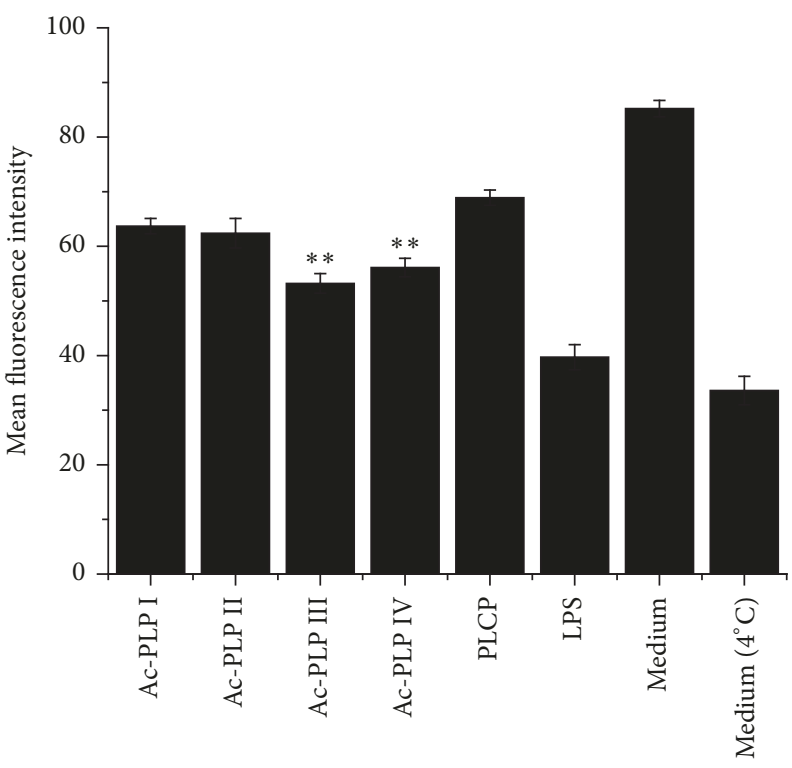

Endocytosis activity of DCs

(b)

Figure 4: Ac-PLCP attenuated the endocytosis activity of DCs. The endocytosis activity was also determined by flow cytometry. The uptake of FITC-dextran of DCs in medium group was extremely high. However, endocytosis activity was significantly attenuated by PLCP treatment. In particular, DCs in Ac-PLCP III and IV groups exhibited much weaker uptake of FITC-dextran. The results shown were from one representative experiment of three independent experiments performed. ${ }^{* *} P<0.01$ compared to the PLCP group.

3.4. Ac-PLCP Increased Expressions of CCR7 and CXCR4 Chemokine mRNA in DCs. The mRNA expression levels of chemokine receptors CCR7 and CXCR4 were analyzed using reverse transcription PCR to investigate the migration capability of DCs. As shown in Figure 5, notable increase was observed in the expressions of both CCR7 and CXCR4 mRNA in Ac-PLCP III and Ac-PLCP IV groups. In particular, the chemokine mRNA expression level in Ac-PLCP III or IV group was significantly higher than PLCP group $(P<0.01)$.
3.5. Ac-PLCP Treated DCs Stimulated T Lymphocyte Proliferation. Splenic T lymphocyte was purified and cocultured with DCs at a ratio of $10: 1$ for $48 \mathrm{~h}$, and then the proliferation of T cells was determined by MTT assay. The proliferation index was calculated as absorbance ratio of polysaccharide group to control group. As shown in Figure 6, compared with control group, the proliferation index was significantly increased in all the polysaccharide groups $(P<0.01)$. The proliferation indexes in Ac-PLCP I and Ac-PLCP II groups were $124.21 \%$ 


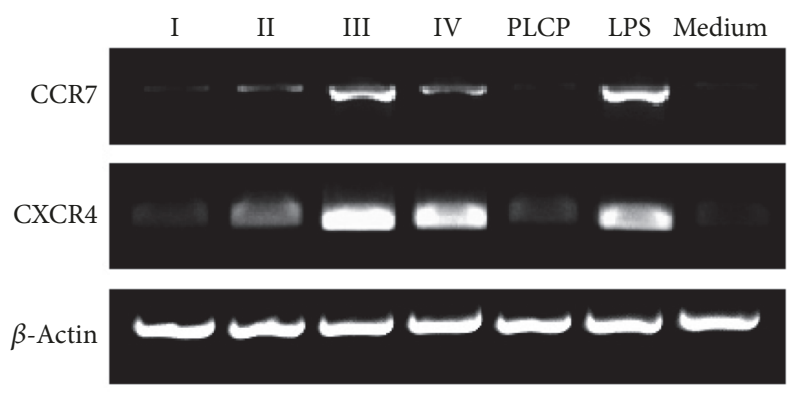

(a)

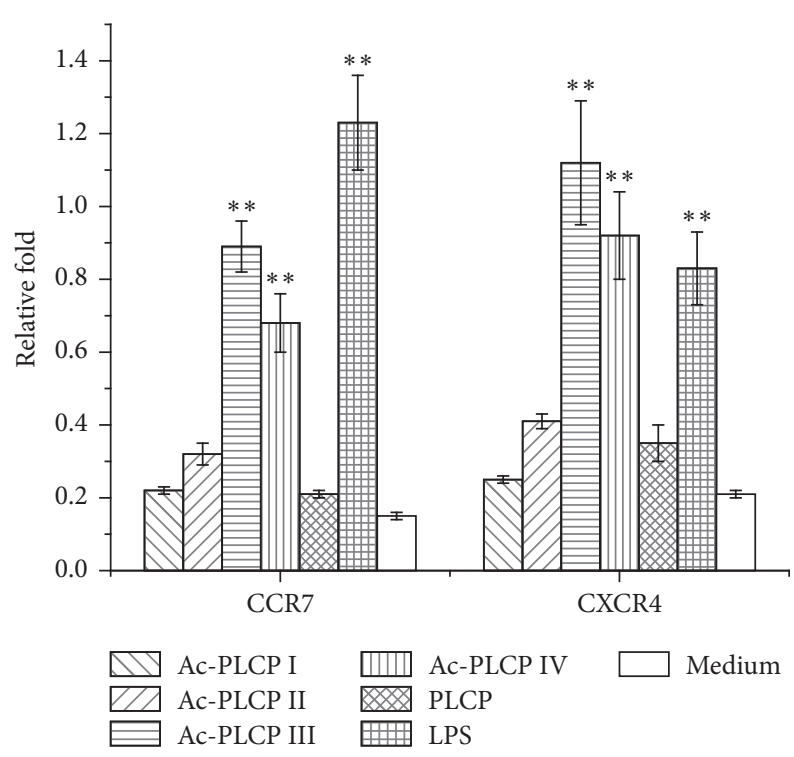

(b)

FIgURE 5: mRNA synthesis levels of CCR7 and CXCR4 were determined through reverse transcriptase PCR. The PCR product was analyzed by $1.5 \%$ agarose gel electrophoresis. The mRNA expression levels of both CCR7 and CXCR4 were significantly increased in Ac-PLCP III and IV groups. Besides, the expression of $\beta$-actin in each group was determined as internal control, and there was no significant difference among all the groups. ${ }^{* *} P<0.01$ compared with PLCP group.

and $146.52 \%$. And the proliferation indexes in Ac-PLCP III and IV groups were $192.75 \%$ and $196.81 \%$, respectively, which were dramatically higher than that in PLCP group (133.62\%) $(P<0.01)$.

\section{Discussion}

DCs are professional antigen-presenting cells and essential mediators of innate and adaptive immune response, as well as tolerance [21]. Immature DCs are strategically located in dermis or mucosal system to capture antigen and then transport these antigens to regional lymph nodes, where they develop into mature and activate lymphocyte to initial immune response. However, immature DCs can weakly activate naïve $\mathrm{T}$ lymphocyte for lacking of antigen-presenting molecules. MHC molecules T cell receptor interaction and CD80(B71)/CD86(B7-2)-CD28 surface molecules interaction between

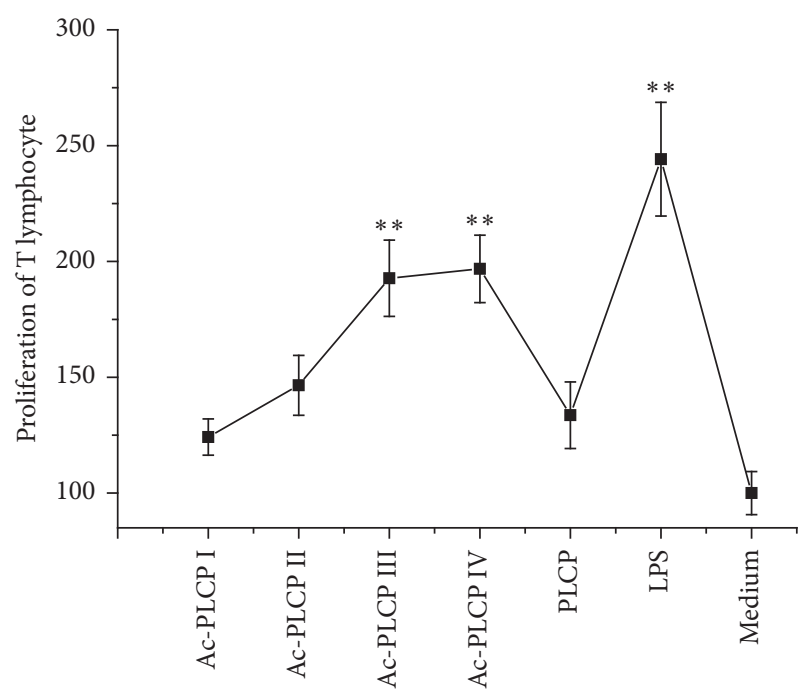

FIGURE 6: DCs treated with Ac-PLCPs showed stronger activity on stimulating $\mathrm{T}$ lymphocyte proliferation. $\mathrm{T}$ lymphocytes were obtained from C57BL/6 $\left(\mathrm{H}-2 \mathrm{~K}^{\mathrm{d}}\right.$ and I- $\left.\mathrm{A}^{\mathrm{d}}\right)$ mice and cocultured with DCs in 96-well plate at the ratio of $10: 1$ for $48 \mathrm{~h}$. The absorption value of the medium group was set at control values in the calculation of $\%$ proliferation. All the polysaccharide-treated DCs showed extremely strong $\mathrm{T}$ cell proliferation stimulating activity. Compared with PLCP group, DCs in Ac-PLCPs III and IV exhibited significantly enhanced T cell proliferation stimulating activity. ${ }^{* *} \mathrm{P}<$ 0.01 compared to the PLCP group.

APCs and $\mathrm{T}$ cells have been regarded as a key point to induce $\mathrm{T}$ cell activation [22]. Though immature dendritic cells synthesize large amounts of MHC II molecules, such MHC II molecules form $\alpha \beta$-dimers that exhibit intracellular distributions. The $\alpha \beta$-dimers are targeted to late endosomes and lysosomes where they reside unproductively with internalized antigens; however, after exposure to inflammatory mediators or microbial products, the newly formed immunogenic MHC II complexes are transported from lysosomes to plasma membrane [23]. In particular, the half-life of MHC II molecules increases from about $10 \mathrm{~h}$ to over $100 \mathrm{~h}$ during this process [24]. Ac-PLCPs III and IV were more effective in inducing DCs maturation than PLCP, since the expressions of MHC II molecules on DCs of the Ac-PLCP III and IV groups were significantly higher than that of PLCP group. Besides the primary signal delivered to T cells through MHC II molecules, the B7-CD28 interaction is one of the dominant costimulatory signals. Particularly, for naïve T cells that express only limited number of costimulatory receptors, CD28 is an indispensable receptor required for T cell priming [25]. The B7-CD28 costimulation signals could direct the development and function of T cells. Lenschow et al. found that effector and regulatory $\mathrm{T}$ cell responses were impaired in mice lacking CD28 or in mice lacking CD80 and CD86. [26]. Gimmi et al. reported that antigen presentation in absence of B7 costimulation results in human T cell clonal anergy [27]. We found that Ac-PLCP III and IV incubation significantly promoted the expression of CD86 molecules on DCs as well, which strongly suggests that the acetylated polysaccharides 
could induce phenotypic maturation on DCs. Meanwhile, an obvious decline of endocytosis activity on DCs was also observed, which indicates that the immature dendritic cells transform from antigen-capturing cells into the mature antigen-presenting cells.

Besides the ligand-receptor interaction, DCs could regulate the polarization of $\mathrm{T}$ lymphocytes by secreting various cytokines. IL-12 is a proinflammatory molecule produced primarily by monocytes/macrophages and DCs. And IL12 principally activates natural killer cells and induces the differentiation of native $\mathrm{CD} 4^{+} \mathrm{T}$ cells to become interferon- $\gamma$ producing T helper 1 (Th1) effectors in cell-mediated immune responses [28]. IL-12 could also synergize with B7/CD28 interaction in inducing efficient proliferation and cytokine production of human T cells [29]. It was reported that CD1c ${ }^{+}$ myeloid DC but not plasmacytoid DC (pDC) in humans can induce high level of cytotoxic molecules in naïve $\mathrm{T}$ cells by producing high amount of IL-12 [30]. The secretion of IL-12p70 of DCs was promoted by Ac-PLPC III and IV treatments, and the levels of IL-12p70 secretion in these two groups were significantly higher than PLCP group. Consistently, we also found that DCs incubated with Ac-PLPC III or IV could significantly stimulate proliferation of naïve $T$ cell in vitro. Furthermore, DCs in Ac-PLCP III and IV groups showed an extremely strong effect on provoking sizeable mixed lymphocyte reaction when cocultured with splenic $\mathrm{T}$ cells, which indicates that the acetylated polysaccharides stimulated functional maturation on DCs. All these findings support that DCs treated with Ac-PLCPs III and IV may possess much more powerful immune regulatory activity than the natural polysaccharide.

Optimal encounter with naïve T cells for the presentation of antigens requires DCs to migrate to secondary lymphoid organs, which is governed by chemokine. The key chemokines directing DCs migration are chemokine (C-C motif) ligand 19 (CCL19), CCL21, and chemokine (C-X-C motif) ligand 12 (CXCL12) [31, 32]. As receptor for CCL19 and CCL21, CC chemokine receptor CCR7 has been found to mediate the migration of DCs from skin into lymphatic vessels [33]. However, the migration of skin DC was initiated by CXCL12-CXCR4 engagement [34]. For plasmacytoid DCs, the migration into splenic white pulp was regulated by CCR7 signal coordinate with CXCR4 signal [35]. And the chemokine signal also enhanced DCs maturation and survival [36]. The migration capability of DCs was enhanced by Ac-PLCP III and IV treatments evidenced by the increased mRNA expression levels of CCR7 and CXCR4.

Dendritic cells are equipped with a battery of patternrecognition receptors (PRRs) that can detect molecular patterns of invading microorganisms or endogenous signals and alter the immune response. The most widely studied family of PRRs on DCs is Toll-like receptors. In particular, TLR4 expressed on the surface of DCs was demonstrated to be one to recognize polysaccharides $[37,38]$. DCs in intestinal mucosa tissue were found to be able to open the tight junctions between epithelial cells without breaking the integrity of the epithelial barrier [39], which provides a probable route for the polysaccharide to interact with DCs. We have also found that TLR- 4 mediates the maturation of DCs induced by PLCP [16]. The acetylation modification may enhance the immunoregulatory activities of PLCP by improving the solubility of PLCP and decreasing the viscosity of the polysaccharide.

In conclusion, Ac-PLCPs exhibited higher maturationstimulating activities on DCs compared with the natural polysaccharide. In particular, the Ac-PLCPs of DS ranging from 0.06 to 0.1 showed the best immunoregulatory activities. The current study also provided a new insight into the structure-activity relationship of PLCP and the utilization of the seeds of Plantago asiatica L.

\section{Abbreviations}

CD: Cluster of differentiation

Ac: Acetylated

DCs: Dendritic cells

DS: Degree of substitution

MHC: Major histocompatibility complex

PLCP: Plantago asiatica L. crude polysaccharide

IL-12: Interleukin-12.

\section{Conflicts of Interest}

The authors declare that there are no conflicts of interest.

\section{Acknowledgments}

This study is supported by the National Key Technology R\&D Program of China (2012BAD33B06), the National Natural Science Foundation of China (21062012, 31260364), and the Program for New Century Excellent Talents in University (NCET-12-0749), which are gratefully acknowledged.

\section{References}

[1] I. Cumpstey, "Chemical Modification of Polysaccharides," ISRN Organic Chemistry, vol. 2013, Article ID 417672, 27 pages, 2013.

[2] D. A. Silva, R. C. M. De Paula, J. P. A. Feitosa, A. C. F. De Brito, J. S. Maciel, and H. C. B. Paula, "Carboxymethylation of cashew tree exudate polysaccharide," Carbohydrate Polymers, vol. 58, no. 2, pp. 163-171, 2004.

[3] J. Xu, W. Liu, W. Yao, X. Pang, D. Yin, and X. Gao, "Carboxymethylation of a polysaccharide extracted from Ganoderma lucidum enhances its antioxidant activities in vitro," Carbohydrate Polymers, vol. 78, no. 2, pp. 227-234, 2009.

[4] J. Wang, L. Zhang, Y. Yu, and P. C. K. Cheung, "Enhancement of antitumor activities in sulfated and carboxymethylated polysaccharides of Ganoderma lucidum," Journal of Agricultural and Food Chemistry, vol. 57, no. 22, pp. 10565-10572, 2009.

[5] L. Ma, H. Chen, Y. Zhang, N. Zhang, and L. Fu, "Chemical modification and antioxidant activities of polysaccharide from mushroom Inonotus obliquus," Carbohydrate Polymers, vol. 89, no. 2, pp. 371-378, 2012.

[6] X.-X. Liu, Z.-J. Wan, L. Shi, and X.-X. Lu, "Preparation and antiherpetic activities of chemically modified polysaccharides from Polygonatum cyrtonema Hua," Carbohydrate Polymers, vol. 83, no. 2, pp. 737-742, 2011. 
[7] I. Y. Bae, H. Y. Kim, S. Lee, and H. G. Lee, "Effect of the degree of oxidation on the physicochemical and biological properties of Grifola frondosa polysaccharides," Carbohydrate Polymers, vol. 83, no. 3, pp. 1298-1302, 2011.

[8] J.-H. Wang, J.-P. Luo, and X.-Q. Zha, "Structural features of a pectic polysaccharide from the stems of Dendrobium nobile Lindl," Carbohydrate Polymers, vol. 81, no. 1, pp. 1-7, 2010.

[9] X. Xing, S. W. Cui, S. Nie, G. O. Phillips, H. D. Goff, and Q. Wang, "Study on Dendrobium officinale O-acetyl-glucomannan (Dendronan ${ }^{\circledR}$ ): part I. Extraction, purification, and partial structural characterization," Bioactive Carbohydrates and Dietary Fibre, vol. 4, no. 1, pp. 74-83, 2014.

[10] H. Qi, Q. Zhang, T. Zhao, R. Hu, K. Zhang, and Z. Li, "In vitro antioxidant activity of acetylated and benzoylated derivatives of polysaccharide extracted from Ulva pertusa (Chlorophyta)," Bioorganic \& Medicinal Chemistry Letters, vol. 16, no. 9, pp. 2441-2445, 2006.

[11] J. Wang, L. Liu, Q. Zhang, Z. Zhang, H. Qi, and P. Li, "Synthesized oversulphated, acetylated and benzoylated derivatives of fucoidan extracted from Laminaria japonica and their potential antioxidant activity in vitro," Food Chemistry, vol. 114, no. 4, pp. 1285-1290, 2009.

[12] Z. Zhang, X. Wang, S. Yu, L. Yin, M. Zhao, and Z. Han, "Synthesized oversulfated and acetylated derivatives of polysaccharide extracted from Enteromorpha linza and their potential antioxidant activity," International Journal of Biological Macromolecules, vol. 49, no. 5, pp. 1012-1015, 2011.

[13] J. Y.-Y. Chan, E. Chan, S.-W. Chan et al., "Enhancement of in vitro and in vivo anticancer activities of polysaccharide peptide from Grifola frondosa by chemical modifications," Pharmaceutical Biology, vol. 49, no. 11, pp. 1114-1120, 2011.

[14] T. Teruya, H. Tatemoto, T. Konishi, and M. Tako, "Structural characteristics and in vitro macrophage activation of acetyl fucoidan from Cladosiphon okamuranus," Glycoconjugate Journal, vol. 26, no. 8, pp. 1019-1028, 2009.

[15] J. Yin, H. Lin, J. Li et al., "Structural characterization of a highly branched polysaccharide from the seeds of Plantago asiatica L.," Carbohydrate Polymers, vol. 87, no. 4, pp. 2416-2424, 2012.

[16] D. Huang, S. Nie, L. Jiang, and M. Xie, "A novel polysaccharide from the seeds of Plantago asiatica L. Induces dendritic cells maturation through toll-like receptor 4," International Immunopharmacology, vol. 18, no. 2, pp. 236-243, 2014.

[17] J.-Y. Yin, S.-P. Nie, C. Zhou, Y. Wan, and M.-Y. Xie, "Chemical characteristics and antioxidant activities of polysaccharide purified from the seeds of Plantago asiatica L," Journal of the Science of Food and Agriculture, vol. 90, no. 2, pp. 210-217, 2010.

[18] Y. Chen, H. Zhang, Y. Wang, S. Nie, C. Li, and M. Xie, "Acetylation and carboxymethylation of the polysaccharide from Ganoderma atrum and their antioxidant and immunomodulating activities," Food Chemistry, vol. 156, pp. 279-288, 2014.

[19] A. B. Das, G. Singh, S. Singh, and C. S. Riar, "Effect of acetylation and dual modification on physico-chemical, rheological and morphological characteristics of sweet potato (Ipomoea batatas) starch," Carbohydrate Polymers, vol. 80, no. 3, pp. 725$732,2010$.

[20] L.-M. Jiang, S.-P. Nie, H.-L. Zhou, D.-F. Huang, and M.-Y. Xie, "Carboxymethylation enhances the maturation-inducing activity in dendritic cells of polysaccharide from the seeds of Plantago asiatica L.," International Immunopharmacology, vol. 22, no. 2, pp. 324-331, 2014.
[21] J. Banchereau and R. M. Steinman, "Dendritic cells and the control of immunity," Nature, vol. 392, no. 6673, pp. 245-252, 1998.

[22] M. Girndt, H. Köhler, E. Schiedhelm-Weick, K.-H. Meyer Zum Büschenfelde, and B. Fleischer, "T cell activation defect in hemodialysis patients: Evidence for a role of the B7/CD28 pathway," Kidney International, vol. 44, no. 2, pp. 359-365, 1993.

[23] A. Chow, D. Toomre, W. Garrett, and I. Mellman, "Dendritic cell maturation triggers retrograde MHC class II transport from lysosomes to the plasma membrane," Nature, vol. 418, no. 6901, pp. 988-994, 2002.

[24] M. Cella, A. Engering, V. Pinet, J. Pieters, and A. Lanzavecchia, "Inflammatory stimuli induce accumulation of MHC class II complexes on dendritic cells," Nature, vol. 388, no. 6644, pp. 782-787, 1997.

[25] L. Chen and D. B. Flies, "Molecular mechanisms of T cell costimulation and co-inhibition," Nature Reviews Immunology, vol. 13, no. 4, pp. 227-242, 2013.

[26] D. J. Lenschow, T. L. Walunas, and J. A. Bluestone, "CD28/B7 system of T cell costimulation," Annual Review of Immunology, vol. 14, pp. 233-258, 1996.

[27] C. D. Gimmi, G. J. Freeman, J. G. Gribben, G. Gray, and L. M. Nadler, "Human T-cell clonal anergy is induced by antigen presentation in the absence of B7 costimulation," Proceedings of the National Acadamy of Sciences of the United States of America, vol. 90, no. 14, pp. 6586-6590, 1993.

[28] X. Ma, W. Yan, H. Zheng et al., "Regulation of IL-10 and IL-12 production and function in macrophages and dendritic cells," F1000Research, vol. 4, 2015.

[29] M. Kubin, M. Kamoun, and G. Trinchieri, "Interleukin 12 synergizes with $\mathrm{B} 7 / \mathrm{CD} 28$ interaction in inducing efficient proliferation and cytokine production of human T cells," The Journal of Experimental Medicine, vol. 180, no. 1, pp. 211-222, 1994.

[30] G. Nizzoli, J. Krietsch, A. Weick et al., "Human CD1c ${ }^{+}$dendritic cells secrete high levels of IL-12 and potently prime cytotoxic Tcell responses," Blood, vol. 122, no. 6, pp. 932-942, 2013.

[31] B. G. Ricart, B. John, D. Lee, C. A. Hunter, and D. A. Hammer, "Dendritic cells distinguish individual chemokine signals through CCR7 and CXCR4," The Journal of Immunology, vol. 186, no. 1, pp. 53-61, 2011.

[32] A. M. Platt and G. J. Randolph, "Dendritic Cell Migration Through the Lymphatic Vasculature to Lymph Nodes," Advances in Immunology, vol. 120, pp. 51-68, 2013.

[33] L. Ohl, M. Mohaupt, N. Czeloth et al., "CCR7 governs skin dendritic cell migration under inflammatory and steady-state conditions," Immunity, vol. 21, no. 2, pp. 279-288, 2004.

[34] K. Kabashima, N. Shiraishi, K. Sugita et al., "CXCL12-CXCR4 engagement is required for migration of cutaneous dendritic cells," The American Journal of Pathology, vol. 171, no. 4, pp. 1249-1257, 2007.

[35] E. Umemoto, K. Otani, T. Ikeno et al., "Constitutive plasmacytoid dendritic cell migration to the splenic white pulp is cooperatively regulated by CCR7- and CXCR4-mediated signaling," The Journal of Immunology, vol. 189, no. 1, pp. 191-199, 2012.

[36] K. Kabashima, K. Sugita, N. Shiraishi, H. Tamamura, N. Fujii, and Y. Tokura, "CXCR4 engagement promotes dendritic cell survival and maturation," Biochemical and Biophysical Research Communications, vol. 361, no. 4, pp. 1012-1016, 2007.

[37] H. S. Kim, B. R. Shin, H. K. Lee et al., "Dendritic cell activation by polysaccharide isolated from Angelica dahurica," Food and Chemical Toxicology, vol. 55, pp. 241-247, 2013. 
[38] M. J. Park, H. S. Ryu, J. S. Kim et al., "Platycodon grandiflorum polysaccharide induces dendritic cell maturation via TLR4 signaling," Food and Chemical Toxicology, vol. 72, pp. 212-220, 2014.

[39] M. Rescigno, M. Urbano, B. Valzasina et al., "Dendritic cells express tight junction proteins and penetrate gut epithelial monolayers to sample bacteria," Nature Immunology, vol. 2, no. 4, pp. 361-367, 2001. 

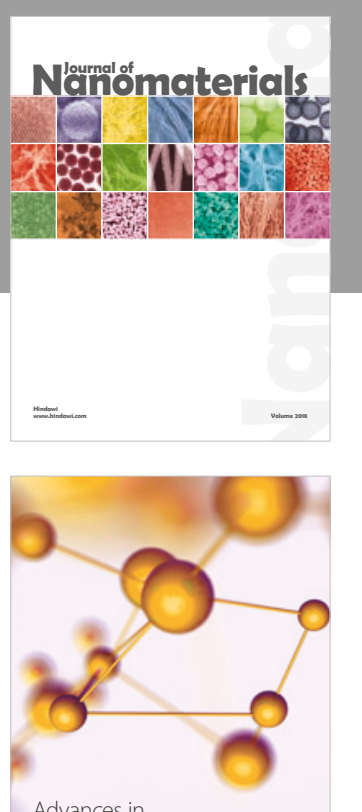

Physical Chemistry
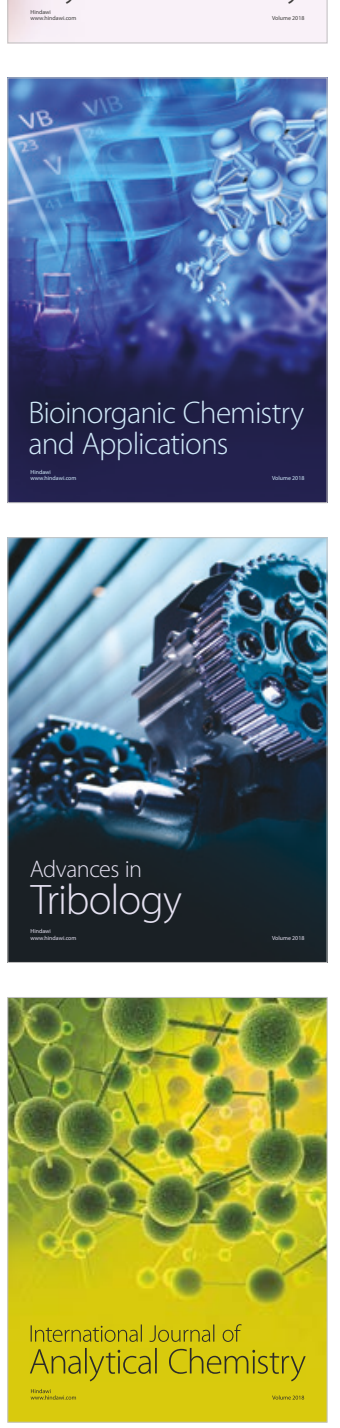

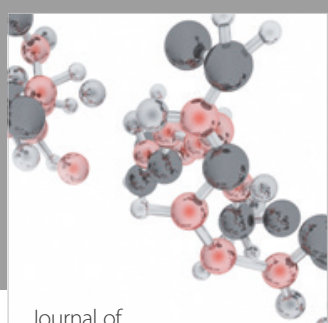

Analytical Methods

in Chemistry

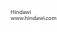

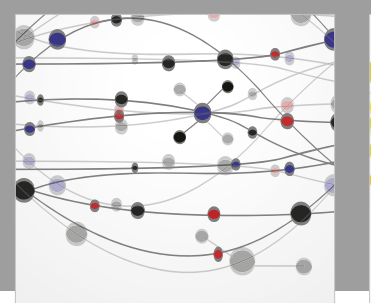

The Scientific World Journal

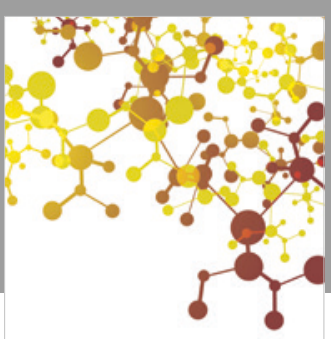

Journal of

Applied Chemistry
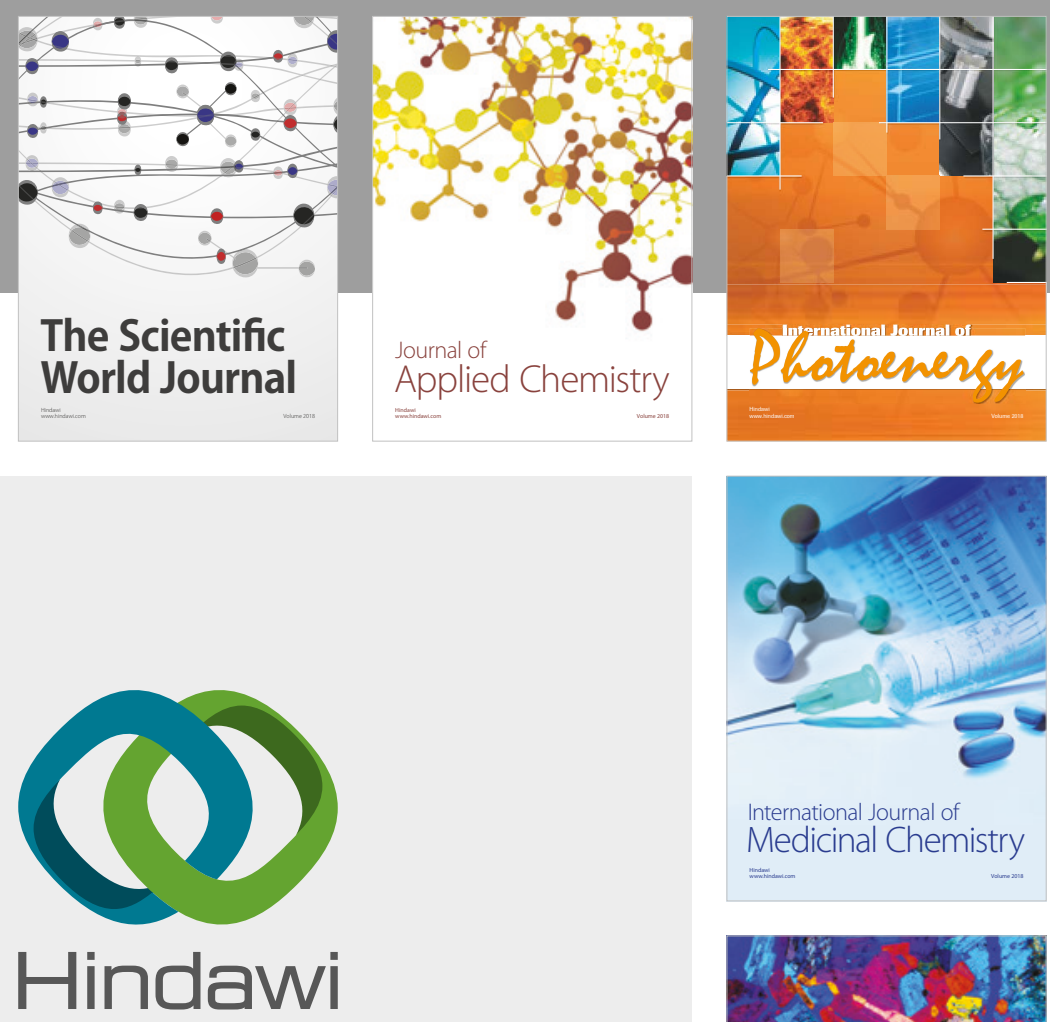

Submit your manuscripts at

www.hindawi.com
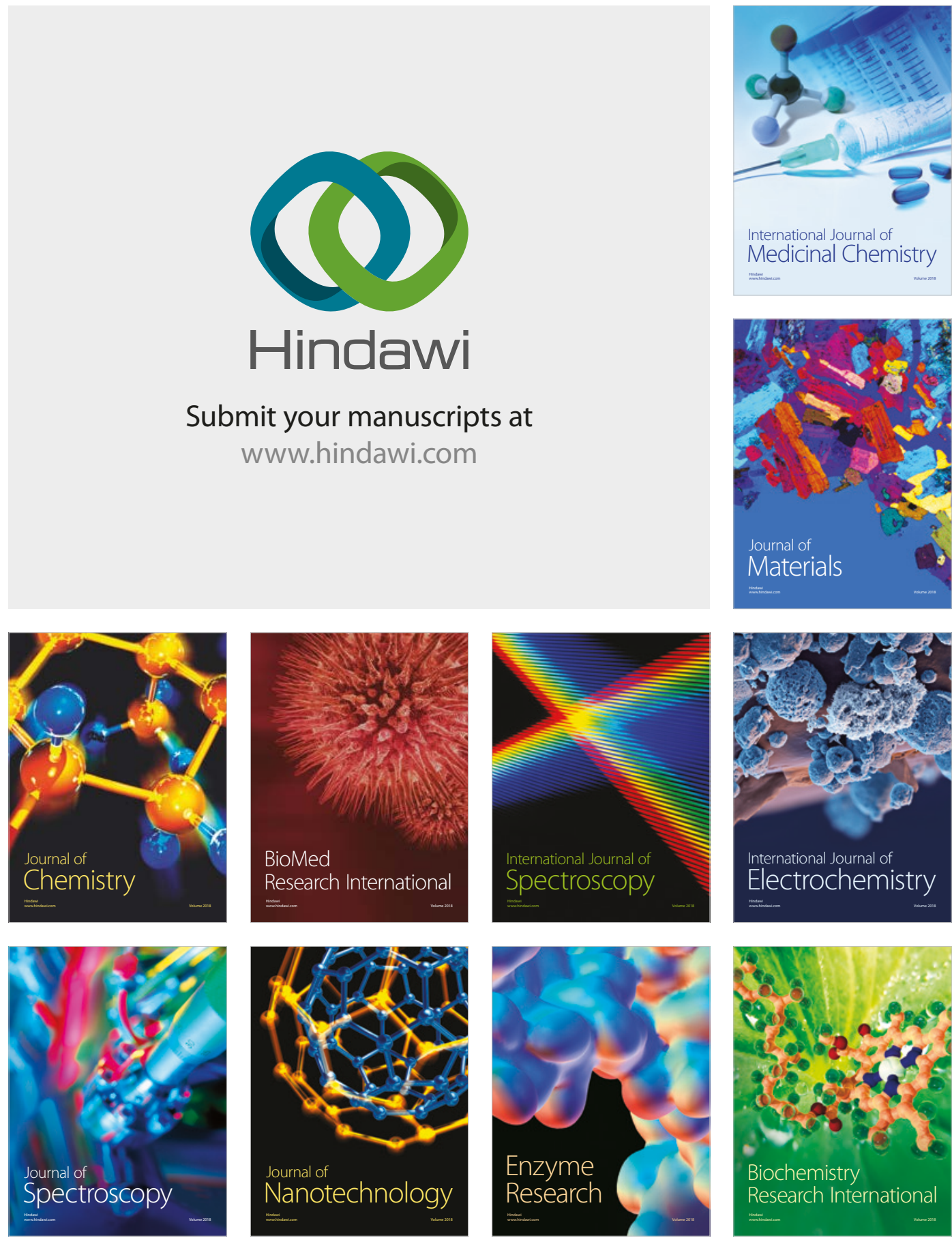
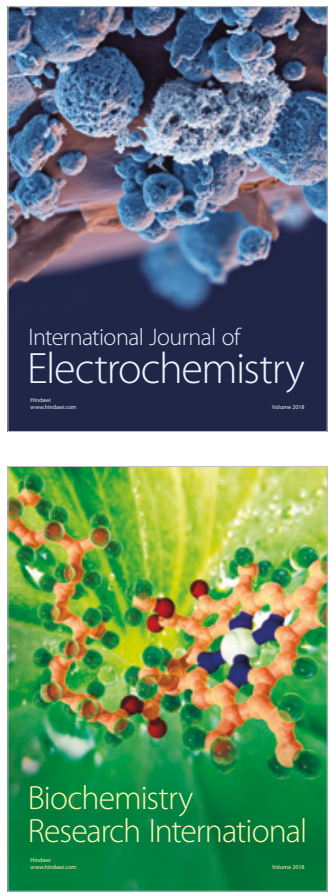